\title{
Reducing the Amount of Pivoting in Symmetric Indefinite Systems
}

\author{
Dulceneia Becker ${ }^{1}$, Marc Baboulin ${ }^{4}$, and Jack Dongarra ${ }^{1,2,3}$ \\ 1 University of Tennessee, USA \\ [dbecker7, dongarra] @eecs.utk.edu \\ 2 Oak Ridge National Laboratory, USA \\ 3 University of Manchester, United Kingdom \\ 4 INRIA / Université Paris-Sud, France \\ marc.baboulin@inria.fr
}

\begin{abstract}
This paper illustrates how the communication due to pivoting in the solution of symmetric indefinite linear systems can be reduced by considering innovative approaches that are different from pivoting strategies implemented in current linear algebra libraries. First a tiled algorithm where pivoting is performed within a tile is described and then an alternative to pivoting is proposed. The latter considers a symmetric randomization of the original matrix using the so-called recursive butterfly matrices. In numerical experiments, the accuracy of tile-wise pivoting and of the randomization approach is compared with the accuracy of the Bunch-Kaufman algorithm.
\end{abstract}

Keywords: dense linear algebra, symmetric indefinite systems, $\mathrm{LDL}^{\mathrm{T}}$ factorization, pivoting, tiled algorithms, randomization.

\section{Introduction}

A symmetric matrix $A$ is called indefinite when the quadratic form $x^{T} A x$ can take on both positive and negative values. By extension, a linear system $A x=b$ is called symmetric indefinite when $A$ is symmetric indefinite. These types of linear systems are commonly encountered in optimization problems coming from physics of structures, acoustics, and electromagnetism, among others. Symmetric indefinite systems also result from linear least squares problems when they are solved via the augmented system method [7, p. 77].

To ensure stability in solving such linear systems, the classical method used is called the diagonal pivoting method [9] where a block-LDL ${ }^{T}$ factorization ${ }^{5}$ is obtained such as

$$
P A P^{T}=L D L^{T}
$$

where $P$ is a permutation matrix, $A$ is a symmetric square matrix, $L$ is unit lower triangular and $D$ is block-diagonal, with blocks of size $1 \times 1$ or $2 \times 2$;

${ }^{5}$ Another factorization method is for example the Aasen's method [13, p.163]: $P A P^{T}=L T L^{T}$ where $L$ is unit lower triangular and $T$ is tridiagonal. 
all matrices are of size $n \times n$. If no pivoting is applied, i.e. $P=I, D$ becomes diagonal. The solution $x$ can be computed by successively solving the triangular or block-diagonal systems $L z=P b, D w=z, L^{T} y=w$, and ultimately we have $x=P^{T} y$.

There are several pivoting techniques that can be applied to determine $P$. These methods involve different numbers of comparisons to find the pivot and have various stability properties. As for the LU factorization, the complete pivoting method (also called Bunch-Parlett algorithm [9]) is the most stable pivoting strategy. It guarantees a satisfying growth factor bound [14, p. 216] but also requires up to $\mathcal{O}\left(n^{3}\right)$ comparisons. The well-known partial pivoting method, based on the Bunch-Kaufman algorithm [8], is implemented in LAPACK [1] and requires at each step of the factorization the exploration of two columns, resulting in a total of $\mathcal{O}\left(n^{2}\right)$ comparisons. This algorithm has good stability properties [14, p. 219] but in certain cases $\|L\|$ may be unbounded, which is a cause for possible instability [3], leading to a modified algorithm referred to as rook pivoting or bounded Bunch-Kaufman pivoting. The latter involves between $\mathcal{O}\left(n^{2}\right)$ and $\mathcal{O}\left(n^{3}\right)$ comparisons depending on the number of $2 \times 2$ pivots. Another pivoting strategy, called Fast Bunch-Parlett strategy (see [3, p. 525] for a description of the algorithm), searches for a local maximum in the current lower triangular part. It is as stable as the rook pivoting but it also requires between $\mathcal{O}\left(n^{2}\right)$ and $\mathcal{O}\left(n^{3}\right)$ comparisons.

With the advent of architectures such as multicore processors [19] and Graphics Processing Unit (GPU), the growing gap between communication and computation efficiency made the communication overhead due to pivoting more critical. These new architectures prompted the need for developing algorithms that lend themselves to parallel execution. A class of such algorithms for shared memory architectures, called Tiled Algorithms, has been developed for one-sided dense factorizations ${ }^{6}[10,11]$ and made available as part of the PLASMA library [12].

Tiled algorithms are based on decomposing the computation in small tasks in order to overcome the intrinsically sequential nature of dense linear algebra methods. These tasks can be executed out of order, as long as dependencies are observed, rendering parallelism. Furthermore, tiled algorithms make use of a tile data-layout where data is stored in contiguous blocks, which differs from the column-wise layout used by LAPACK, for instance. The tile data-layout allows the computation to be performed on small blocks of data that fit into cache, and hence exploits cache locality and re-use. However, it does not lend itself straightforwardly for pivoting, as this requires a search for pivots and permutations over full columns/rows. For symmetric matrices, the difficulties are even greater since symmetric pivoting requires interchange of both rows and columns. The search for pivots outside a given tile curtails memory locality and data dependence between tiles (or tasks). The former has a direct impact on the performance of serial kernels and the latter on parallel performance (by increasing data dependence among tiles, granularity is decreased and therefore scalability) [18].

\footnotetext{
${ }^{6} \mathrm{LDL}^{\mathrm{T}}$ is still under development and shall be available in the future [6].
} 
In this paper, the possibility of eliminating the overhead due to pivoting by considering randomization techniques is also investigated. These techniques were initially proposed in [16] and modified approaches were studied in $[4,5]$ for the LU factorization. In this context, they are applied to the case of symmetric indefinite systems. According to this random transformation, the original matrix $A$ is transformed into a matrix that would be sufficiently "random" so that, with a probability close to 1 , pivoting is not needed. This transformation is a multiplicative preconditioning by means of random matrices called recursive butterfly matrices. The $\mathrm{LDL}^{\mathrm{T}}$ factorization without pivoting is then applied to the preconditioned matrix. One observes that two levels of recursion for butterfly matrices are enough to obtain an accuracy close to that of $\mathrm{LDL}^{\mathrm{T}}$ with either partial (Bunch-Kaufman) or rook pivoting on a collection of matrices. The overhead is reduced to $\sim 8 n^{2}$ operations, which is negligible when compared to the cost of pivoting.

\section{Tile-wise Pivoting}

Given Equation (1), the tiled algorithm starts by decomposing $A$ in $n t \times n t$ tiles $^{7}$ (blocks), where each $A_{i j}$ is a tile of size $m b \times n b$. The same decomposition can be applied to $L$ and $D$. For instance, for $n t=3$ :

$$
\left[\begin{array}{lll}
A_{11} & A_{12} & A_{13} \\
A_{21} & A_{22} & A_{23} \\
A_{31} & A_{32} & A_{33}
\end{array}\right]=\left[\begin{array}{lll}
L_{11} & & \\
L_{21} & L_{22} & \\
L_{31} & L_{32} & L_{33}
\end{array}\right]\left[\begin{array}{lll}
D_{11} & & \\
& D_{22} & \\
& & D_{33}
\end{array}\right]\left[\begin{array}{lll}
L_{11}^{T} & L_{21}^{T} & L_{31}^{T} \\
& L_{22}^{T} & L_{32}^{T} \\
& & L_{33}^{T}
\end{array}\right]
$$

Upon this decomposition and using the same principle as the Schur complement, a series of tasks can be set to calculate each $L_{i j}$ and $D_{i i}$ :

$$
\begin{aligned}
{\left[L_{11}, D_{11}\right] } & =\operatorname{LDL}\left(A_{11}\right) \\
L_{21} & =A_{12}\left(D_{11} L_{11}^{T}\right)^{-1} \\
L_{31} & =A_{13}\left(D_{11} L_{11}^{T}\right)^{-1} \\
\tilde{A}_{22} & =A_{22}-L_{21} D_{11} L_{21}^{T} \\
{\left[L_{22}, D_{22}\right] } & =\operatorname{LDL}\left(\tilde{A}_{22}\right) \\
\tilde{A}_{32} & =A_{32}-L_{31} D_{11} L_{21}^{T} \\
L_{32} & =\tilde{A}_{32}\left(D_{22} L_{22}^{T}\right)^{-1} \\
\tilde{A}_{33} & =A_{33}-L_{31} D_{11} L_{31}^{T}-L_{32} D_{22} L_{32}^{T} \\
{\left[L_{33}, D_{33}\right] } & =\operatorname{LDL}\left(\tilde{A}_{33}\right)
\end{aligned}
$$

$\operatorname{LDL}\left(X_{k k}\right)$ at Equations (2), (6) and (10) means the actual $\mathrm{LDL}^{\mathrm{T}}$ factorization of tile $X_{k k}$. These tasks can be executed out of order, as long as dependencies are observed, rendering parallelism (see [6] for more details).

\footnotetext{
${ }^{7}$ For rectangular matrices, $A$ is decomposed into $m t \times n t$ tiles.
} 
Following the same approach, for $P A P^{T}=L D L^{T}$, Equation (1), i.e. with pivoting, the tasks for $n t=3$ may be described as:

$$
\begin{aligned}
{\left[L_{11}, D_{11}, P_{11}\right] } & =\operatorname{LDL}\left(A_{11}\right) \\
L_{21} & =P_{22}^{T} A_{21} P_{11}\left(D_{11} L_{11}^{T}\right)^{-1} \\
L_{31} & =P_{33}^{T} A_{31} P_{11}\left(D_{11} L_{11}^{T}\right)^{-1} \\
\tilde{A}_{22} & =A_{22}-\left(P_{22} L_{21}\right) D_{11}\left(P_{22} L_{21}\right)^{T} \\
{\left[L_{22}, D_{22}, P_{22}\right] } & =\operatorname{LDL}\left(\tilde{A}_{22}\right) \\
L_{32} & =P_{33}^{T} \tilde{A}_{32} P_{22}\left(D_{22} L_{22}^{T}\right)^{-1} \\
\tilde{A}_{33} & =A_{33}-\left(P_{33} L_{31}\right) D_{11}\left(P_{33} L_{31}\right)^{T}-\left(P_{33} L_{32}\right) D_{22}\left(P_{33} L_{32}\right)^{T} \\
{\left[L_{33}, D_{33}, P_{33}\right] } & =\operatorname{LDL}\left(\tilde{A}_{33}\right)
\end{aligned}
$$

Equations (11) to (18) are similar to Equations (2) to (10), except that the permutation matrix $P_{k k}$ has been added. This permutation matrix $P_{k k}$ generates a cross-dependence between equations, which is not an issue when pivoting is not used. For instance, in order to calculate

$$
L_{21}=P_{22}^{T} A_{21} P_{11}\left(D_{11} L_{11}^{T}\right)^{-1}
$$

$P_{22}$ is required. However, to calculate

$$
\left[L_{22}, D_{22}, P_{22}\right]=\operatorname{LDL}\left(A_{22}-\left(P_{22} L_{21}\right) D_{11}\left(P_{22} L_{21}\right)^{T}\right)
$$

$L_{21}$ is required. To overcome this cross-dependence, instead of actually calculating $L_{21}, P_{22} L_{21}$ is calculated instead, since the equations can be rearranged such as $P_{22} L_{21}$ is always used and therefore $L_{21}$ is not needed. Hence, Equations (12), (13) and (16) become, in a general form,

$$
P_{i i} L_{i j}=A_{i j} P_{j j}\left(D_{j j} L_{j j}^{T}\right)^{-1}
$$

After $P_{i i}$ is known, $L_{i j}$, for $1 \geq j \geq i-1$, can be calculated such as

$$
L_{i j}=P_{i i}^{T} L_{i j}
$$

This procedure may be described as in Algorithm 1, where $A$ is a symmetric matrix of size $n \times n$ split in $n t \times n t$ tiles $A_{i j}$, each of size $m b \times n b$.

The permutation matrices $P_{k k}$ of Algorithm 1 are computed during the factorization of tile $A_{k k}$. If pivots were searched only inside tile $A_{i i}$, the factorization would depend only and exclusively on $A_{k k}$. However, for most pivoting techniques, pivots are searched throughout columns, which make the design of efficient parallel algorithm very difficult [18].

The tile-wise pivoting restricts the search of pivots to the tile $A_{k k}$ when factorizing it, i.e. if LAPACK [1] routine XSYTRF was chosen to perform the factorization, it could be used as it is. In other words, the same procedure used to factorize an entire matrix $A$ is used to factorize the tile $A_{k k}$. This approach does 


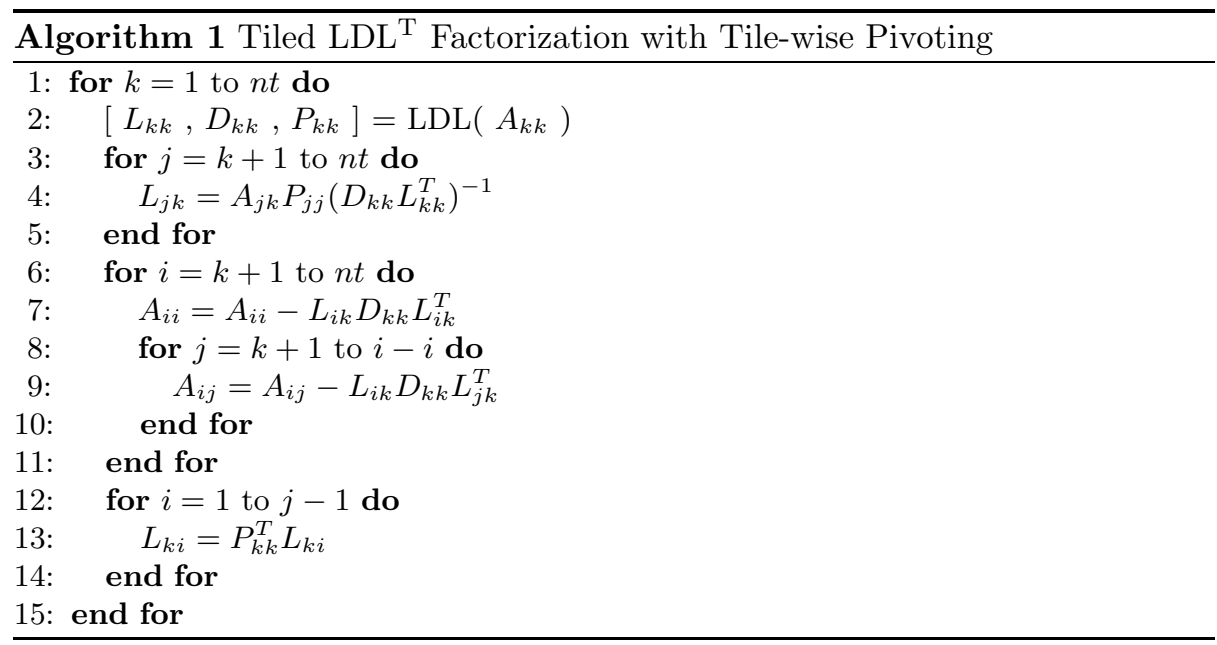

not guarantee the accuracy of the solution; it strongly depends on the matrix to be factorized and how the pivots are distributed. However, it guarantees numerical stability of the factorization of each tile $A_{k k}$, as long as an appropriate pivoting technique is applied. For instance, $\mathrm{LDL}^{\mathrm{T}}$ without pivoting fails as soon as a zero is found on the diagonal, while the tile-wise pivoted $\mathrm{LDL}^{\mathrm{T}}$ does not, as shown in Section 4. Note that pivoting is applied as part of a sequential kernel, which means that the pivot search and hence the permutations are also serial.

\section{An Alternative to Pivoting in Symmetric Indefinite Systems}

A randomization technique that allows pivoting to be avoided in the $\mathrm{LDL}^{\mathrm{T}}$ factorization is described. This technique was initially proposed in [16] in the context of general linear systems where the randomization is referred to as Random Butterfly Transformation (RBT). Then a modified approach has been described in [5] for the LU factorization of general dense matrices and we propose here to adapt this technique specifically to symmetric indefinite systems. It consists of a multiplicative preconditioning $U^{T} A U$ where the matrix $U$ is chosen among a particular class of random matrices called recursive butterfly matrices. Then $\mathrm{LDL}^{\mathrm{T}}$ factorization without pivoting is performed on the symmetric matrix $U^{T} A U$ and, to solve $A x=b,\left(U^{T} A U\right) y=U^{T} b$ is solved instead, followed by $x=U y$. We study the random transformation with recursive butterfly matrices, and minimize the number of recursion steps required to get a satisfying accuracy. The resulting transformation will be called Symmetric Random Butterfly Transformation (SRBT). We define two types of matrices that will be used in the symmetric random transformation. These definitions are inspired from [16] in the particular case of real-valued matrices. 
Definition 1 A butterfly matrix is defined as any $n$-by-n matrix of the form:

$$
B=\frac{1}{\sqrt{2}}\left(\begin{array}{cc}
R_{0} & R_{1} \\
R_{0} & -R_{1}
\end{array}\right)
$$

where $n \geq 2$ and $R_{0}$ and $R_{1}$ are random diagonal and nonsingular $n / 2-b y-n / 2$ matrices.

Definition 2 A recursive butterfly matrix of size $n$ and depth $d$ is a product of the form

$$
\begin{aligned}
W^{<n, d>}= & \left(\begin{array}{ccc}
B_{1}^{<n / 2^{d-1}>} & \cdots & 0 \\
\vdots & \ddots & \vdots \\
0 & \cdots & B_{2^{d-1}}^{<n / 2^{d-1}>}
\end{array}\right) \times \cdots \times \\
& \left(\begin{array}{cccc}
B_{1}^{<n / 4>} & 0 & 0 & 0 \\
0 & B_{2}^{<n / 4>} & 0 & 0 \\
0 & 0 & B_{3}^{<n / 4>} & 0 \\
0 & 0 & 0 & B_{4}^{<n / 4>}
\end{array}\right) \times\left(\begin{array}{cc}
B_{1}^{<n / 2>} & 0 \\
0 & B_{2}^{<n / 2>}
\end{array}\right) \times B^{<n>}
\end{aligned}
$$

where $B_{i}^{<n / 2^{k-1}>}$ are butterfly matrices of size $n / 2^{k-1}$ with $1 \leq k \leq d$.

Note that this definition requires that $n$ is a multiple of $2^{d-1}$ which can always be obtained by "augmenting" the matrix $A$ with additional 1's on the diagonal. Note also that Definition 2 differs from the definition of a recursive butterfly matrix given in [16], which corresponds to the special case where $d=\log _{2} n+1$, i.e. the first term of the product expressing $W^{<n, d>}$ is a diagonal matrix of size $n$.

For instance, if $n=4$ and $d=2$, then the recursive butterfly matrix $W^{<4,2>}$ is defined by

$$
\begin{aligned}
& W^{<4,2>}=\left(\begin{array}{cc}
B_{1}^{<2>} & 0 \\
0 & B_{2}^{<2>}
\end{array}\right) \times B^{<4>} \\
& =\frac{1}{2}\left(\begin{array}{cccc}
r_{1}^{<2>} & r_{2}^{<2>} & 0 & 0 \\
r_{1}^{<2>} & -r_{2}^{<2>} & 0 & 0 \\
0 & 0 & r_{3}^{<2>} & r_{4}^{<2>} \\
0 & 0 & r_{3}^{<2>} & -r_{4}^{<2>}
\end{array}\right)\left(\begin{array}{cccc}
r_{1}^{<4>} & 0 & r_{3}^{<4>} & 0 \\
0 & r_{2}^{<4>} & 0 & r_{4}^{<4>} \\
r_{1}^{<4>} & 0 & -r_{3}^{<4>} & 0 \\
0 & r_{2}^{<4>} & 0 & -r_{4}^{<4>}
\end{array}\right) \\
& =\frac{1}{2}\left(\begin{array}{ccccc}
r_{1}^{<2>} r_{1}^{<4>} & r_{2}^{<2>} r_{2}^{<4>} & r_{1}^{<2>} r_{3}^{<4>} & r_{2}^{<2>} r_{4}^{<4>} \\
r_{1}^{<2>} r_{1}^{<4>} & -r_{2}^{<2>} r_{2}^{<4>} & r_{1}^{<2>} r_{3}^{<4>} & -r_{2}^{<2>} r_{4}^{<4>} \\
r_{3}^{<2>} r_{1}^{<4>} & r_{4}^{<2>} r_{2}^{<4>} & -r_{3}^{<>} r_{3}^{<4>} & -r_{4}^{<2>} r_{4}^{<4>} \\
r_{3}^{<2>} r_{1}^{<4>} & -r_{4}^{<2>} r_{2}^{<4>} & -r_{3}^{<2>} r_{3}^{<4>} & r_{4}^{<2>} r_{4}^{<4>}
\end{array}\right),
\end{aligned}
$$

where $r_{i}^{<j>}$ are real random entries.

The objective here is to minimize the computational cost of the RBT defined in [16] by considering a number of recursions $d$ such that $d \ll n$, resulting in the transformation defined as follows. 
Definition 3 A symmetric random butterfly transformation (SRBT) of depth $d$ of a square matrix $A$ is the product:

$$
A_{r}=U^{T} A U
$$

where $U$ is a recursive butterfly matrix of depth $d$.

Remark 1 Let $A$ be a square matrix of size $n$, the computational cost of a multiplication $B^{T} A B$ with $B$ butterfly of size $n$ is $M(n)=4 n^{2}$. Then the number of operations involved in the computation of $A_{r}$ by an SRBT of depth $d$ is

$$
\begin{aligned}
C(n, d) & =\sum_{k=1}^{d}\left(\left(2^{k-1}\right)^{2} \times M\left(n / 2^{k-1}\right)\right)=\sum_{k=1}^{d}\left(\left(2^{k-1}\right)^{2} \times 4\left(n / 2^{k-1}\right)^{2}\right) \\
& =\sum_{k=1}^{d}\left(4 n^{2}\right)=4 d n^{2}
\end{aligned}
$$

Note that the maximum cost in the case of an RBT as described in [16] is

$$
C\left(n, \log _{2} n+1\right) \simeq 4 n^{2} \log _{2} n .
$$

We can find in [16] details on how RBT might affect the growth factor and in [5] more information concerning the practical computation of $A_{r}$ as well as a packed storage description and a condition number analysis. Note that, since we know that we do not pivot when using SRBT, the LDL ${ }^{T}$ factorization without pivoting can be performed with a very efficient tiled algorithm [6].

\section{Numerical Experiments}

Experiments to measure the accuracy of each procedure described in the previous sections were carried out using Matlab version 7.12 (R2011a) on a machine with a precision of $2.22 \cdot 10^{-16}$. Table 1 presents accuracy comparisons of linear systems solved using the factors of $A$ calculated by $\mathrm{LDL}^{\mathrm{T}}$ with: no pivoting $(\mathrm{NP})$, partial pivoting $(\mathrm{PP})$, tile-wise pivoting $(\mathrm{TP})$, and no pivoting preceded by the Symmetric Random Butterfly Transformation (SRBT).

The partial pivoting corresponds to the Bunch-Kaufman algorithm as it is implemented in LAPACK. Note that for all experiments the rook pivoting method achieves the same accuracy as the partial pivoting and therefore is not listed.

All matrices are of size $1024 \times 1024$, either belonging to the Matlab gallery or the Higham's Matrix Computation Toolbox [14] or generated using Matlab function rand. Matrices $|i-j|, \max (i, j)$ and Hadamard are defined in the experiments performed in [16]. Matrices rand1 and rand2 correspond to random matrices with entries uniformly distributed in $[0,1]$ with all and $1 / 4$ of the diagonal elements set to 0 , respectively. Matrices rand0 and rand4 are also random matrices, where the latter has its diagonal elements scaled by $1 / 1000$.

For all test matrices, we suppose that the exact solution is $x=\left[\begin{array}{lll}1 & 1 \ldots 1\end{array}\right]$ and we set the right-hand side $b=A x$. In Table 1, the 2-norm condition number 
Table 1. Component-wise backward error for $\mathrm{LDL}^{\mathrm{T}}$ solvers on a set of test matrices.

\begin{tabular}{|c|c|c|c|c|c|}
\hline Matrix & Cond $\mathrm{A}$ & $\mathrm{NP}$ & $\mathrm{PP}$ & $\mathrm{TP}$ & SRBT (IR) \\
\hline condex & $1 \cdot 10^{2}$ & $5.57 \cdot 10^{-15}$ & $6.94 \cdot 10^{-15}$ & $7.44 \cdot 10^{-15}$ & $6.54 \cdot 10^{-15}(0)$ \\
\hline fiedler & $7 \cdot 10^{5}$ & Fail & $2.99 \cdot 10^{-15}$ & $7.43 \cdot 10^{-15}$ & $9.37 \cdot 10^{-15}(0)$ \\
\hline orthog & $1 \cdot 10^{0}$ & $8.40 \cdot 10^{-1}$ & $1.19 \cdot 10^{-14}$ & $5.31 \cdot 10^{-1}$ & $3.51 \cdot 10^{-16}(1)$ \\
\hline randcorr & $3 \cdot 10^{3}$ & $4.33 \cdot 10^{-16}$ & $3.45 \cdot 10^{-16}$ & $4.40 \cdot 10^{-16}$ & $5.10 \cdot 10^{-16}(0)$ \\
\hline augment & $5 \cdot 10^{4}$ & $7.70 \cdot 10^{-15}$ & $4.11 \cdot 10^{-15}$ & $8.00 \cdot 10^{-15}$ & $2.59 \cdot 10^{-16}(1)$ \\
\hline prolate & $6 \cdot 10^{18}$ & $8.18 \cdot 10^{-15}$ & $8.11 \cdot 10^{-16}$ & $2.62 \cdot 10^{-15}$ & $2.67 \cdot 10^{-15}(0)$ \\
\hline toeppd & $1 \cdot 10^{7}$ & $5.75 \cdot 10^{-16}$ & $7.75 \cdot 10^{-16}$ & $6.99 \cdot 10^{-16}$ & $2.38 \cdot 10^{-16}(0)$ \\
\hline ris & $4 \cdot 10^{0}$ & Fail & $3.25 \cdot 10^{-15}$ & $8.81 \cdot 10^{-1}$ & $6.05 \cdot 10^{-1}(10)$ \\
\hline$|i-j|$ & $7 \cdot 10^{5}$ & $2.99 \cdot 10^{-15}$ & $2.99 \cdot 10^{-15}$ & $7.43 \cdot 10^{-15}$ & $1.15 \cdot 10^{-14}(0)$ \\
\hline $\max (i, j)$ & $3 \cdot 10^{6}$ & $2.35 \cdot 10^{-14}$ & $2.06 \cdot 10^{-15}$ & $5.08 \cdot 10^{-15}$ & $1.13 \cdot 10^{-14}(0)$ \\
\hline Hadamard & $1 \cdot 10^{0}$ & $0 \cdot 10^{0}$ & $0 \cdot 10^{0}$ & $0 \cdot 10^{0}$ & $7.29 \cdot 10^{-15}(0)$ \\
\hline rand0 & $2 \cdot 10^{5}$ & $1.19 \cdot 10^{-12}$ & $7.59 \cdot 10^{-14}$ & $1.69 \cdot 10^{-13}$ & $1.64 \cdot 10^{-15}(1)$ \\
\hline rand1 & $2 \cdot 10^{5}$ & Fail & $1.11 \cdot 10^{-13}$ & $2.07 \cdot 10^{-11}$ & $1.77 \cdot 10^{-15}(1)$ \\
\hline rand2 & $1 \cdot 10^{5}$ & Fail & $5.96 \cdot 10^{-14}$ & $6.41 \cdot 10^{-13}$ & $1.77 \cdot 10^{-15}(1)$ \\
\hline rand3 & $8 \cdot 10^{4}$ & $4.69 \cdot 10^{-13}$ & $7.60 \cdot 10^{-14}$ & $4.07 \cdot 10^{-13}$ & $1.92 \cdot 10^{-15}(1)$ \\
\hline $\begin{array}{l}\mathrm{LDL}^{\mathrm{T}} \\
\mathrm{LDL}^{\mathrm{T}}\end{array}$ & & & & idom Butterfl & Transformation \\
\hline
\end{tabular}

of each matrix is listed. Note that we also computed the condition number of the randomized matrix which, similarly to [5], is of same order of magnitude as cond $\mathrm{A}$ and therefore is not listed. For each $\mathrm{LDL}^{\mathrm{T}}$ solver, the component-wise backward error is reported. The latter is defined in [15] and expressed as

$$
\omega=\max _{i} \frac{|A \hat{x}-b|_{i}}{(|A| \cdot|\hat{x}|+|b|)_{i}},
$$

where $\hat{x}$ is the computed solution.

Similarly to [16], the random diagonal matrices used to generate the butterfly matrices described in Definition 1 have diagonal values $\exp \left(\frac{r}{10}\right)$ where $r$ is randomly chosen in $\left[-\frac{1}{2}, \frac{1}{2}\right]$ (matlab instruction rand). The number of recursions used in the SRBT algorithm (parameter $d$ in Definition 3) has been set to 2 . Hence, the resulting cost of SRBT is $\sim 8 n^{2}$ operations (see Remark 1). To improve the stability, iterative refinement (in the working precision) is added when SRBT is used. Similarly to $[2,17]$, the iterative refinement algorithm is called while $\omega>(n+1) u$, where $u$ is the machine precision. The number of iterations (IR) in the iterative refinement process is also reported in Table 1.

For all matrices, except orthog and ris with TP and ris with SRBT, the factorization with both tile-wise pivoting and randomization achieves satisfactory results. Iterative refinement turns out to be necessary in a few cases when using SRBT but with never more than one iteration (except for ris for which 
neither TP nor SRBT have achieved accurate results). For matrix prolate, all methods result in a small backward error. However, the solution cannot be accurate at all due to the large condition number. Note that when matrices are orthogonal (orthog) or proportional to an orthogonal matrix (Hadamard), $\mathrm{LDL}^{\mathrm{T}}$ must not be used. Also, toeppd is positive definite and would normally be solved by Cholesky and not $\mathrm{LDL}^{\mathrm{T}}$. These three test cases have been used only for testing purposes. In the case of the integer-valued matrix Hadamard, SRBT destroys the integer structure and transforms the initial matrix into a real-valued one. For the four random matrices, TP achieves results slightly less accurate than SRBT. However, in these cases iterative refinement added to TP would enable us to achieve an accuracy similar to SRBT.

TP and SRBT are always more accurate than NP but they both failed to produce results as accurate as PP for at least one of the test matrices. Nevertheless, despite the reduced number of test cases, they cover a reasonable range of matrices, including those with zeros on the diagonal. Test case rand1 has only zeros on the diagonal and was accurately solved by both techniques. This case fails at the very first step of the $\mathrm{LDL}^{\mathrm{T}}$ method without pivoting. Test case orthog has been solved accurately with SRBT but not with TP. For this particular case, when the pivot search is applied on the full matrix, rows/columns 1 and $n$ are permuted, then rows/columns 2 and $n-1$ are permuted, and so forth. In others, the pivots are spread far apart and the tile-wise pivoting cannot reach them, i.e. there are not good enough pivots within each tile.

\section{Conclusion and Future Work}

A tiled $\mathrm{LDL}^{\mathrm{T}}$ factorization with tile-wise pivoting and a randomization technique to avoid pivoting in the $\mathrm{LDL}^{\mathrm{T}}$ factorization have been presented. The tilewise pivoting consists of choosing a pivoting strategy and restraining the pivot search to the tile being factored. The randomization technique, called Symmetric Random Butterfly Transformation (SRBT), involves a multiplicative preconditioning which is computationally very affordable and negligible compared to the communication overhead due to classical pivoting algorithms.

Both techniques give accurate results on most test cases considered in this paper, including pathological ones. However, further development of the tile-wise pivoting is required in order to increase its robustness. In particular, techniques such as search by pairs of tiles, also called incremental pivoting, have to be investigated for symmetric indefinite factorizations. Also, to improve stability, the solution obtained after randomization should be systematically followed by iterative refinement in fixed precision (one iteration is sufficient in general). The algorithms presented in this paper shall be integrated into PLASMA, which will

allow performance comparisons of the $\mathrm{LDL}^{\mathrm{T}}$ solvers and more extensive testing using the matrices available as part of LAPACK. 


\section{References}

1. E. Anderson, Z. Bai, C. Bischof, S. Blackford, J. Demmel, J. Dongarra, J. D. Croz, A. Greenbaum, S. Hammarling, A. McKenney, and D. Sorensen. LAPACK User's Guide. SIAM, 1999. Third edition.

2. M. Arioli, J. W. Demmel, and I. S. Duff. Solving sparse linear systems with sparse backward error. SIAM J. Matrix Anal. and Appl., 10(2):165-190, 1989.

3. C. Ashcraft, R. G. Grimes, and J. G. Lewis. Accurate symmetric indefinite linear equation solvers. SIAM J. Matrix Anal. and Appl., 20(2):513-561, 1998.

4. M. Baboulin, J. Dongarra, and S. Tomov. Some issues in dense linear algebra for multicore and special purpose architectures. In Proceedings of the 9th International Workshop on State-of-the-Art in Scientific and Parallel Computing (PARA'08).

5. M. Baboulin, J. Dongarra, J. Herrmann, and S. Tomov. Accelerating linear system solutions using randomization techniques. Lapack Working Note 246 and INRIA Research Report 7616, May 2011.

6. D. Becker, M. Faverge, and J. Dongarra. Towards a Parallel Tile LDL Factorization for Multicore Architectures. Technical Report ICL-UT-11-03, Innovative Computing Laboratory, University of Tennessee, Knoxville, TN, USA, April 2011.

7. Å. Björck. Numerical Methods for Least Squares Problems. Society for Industrial and Applied Mathematics, 1996.

8. J. R. Bunch and L. Kaufman. Some stable methods for calculating inertia and solving symmetric linear systems. Math. Comput., 31:163-179, 1977.

9. J. R. Bunch and B. N. Parlett. Direct methods for solving symmetric indefinite systems of linear equations. SIAM J. Numerical Analysis, 8:639-655, 1971.

10. A. Buttari, J. Langou, J. Kurzak, and J. Dongarra. Parallel tiled QR factorization for multicore architectures. Concurrency Computat.: Pract. Exper., 20(13):15731590, 2008.

11. A. Buttari, J. Langou, J. Kurzak, and J. Dongarra. A class of parallel tiled linear algebra algorithms for multicore architectures. Parallel Comput. Syst. Appl., 35:3853, 2009.

12. J. Dongarra, J. Kurzak, J. Langou, J. Langou, H. Ltaief, P. Luszczek, A. YarKhan, W. Alvaro, M. Faverge, A. Haidar, J. Hoffman, E. Agullo, A. Buttari, and B. Hadri. PLASMA Users' Guide, Version 2.3. Technical Report, Electrical Engineering and Computer Science Department, Univesity of Tennessee, Knoxville, TN, Sep 2010.

13. G. H. Golub and C. F. van Loan. Matrix Computations. The Johns Hopkins University Press, 1996. Third edition.

14. N. J. Higham. Accuracy and Stability of Numerical Algorithms. SIAM, 2002. Second edition.

15. W. Oettli and W. Prager. Compatibility of approximate solution of linear equations with given error bounds for coefficients and right-hand sides. Numerische Mathematik, 6:405-409, 1964.

16. D. S. Parker. Random butterfly transformations with applications in computational linear algebra. Technical Report CSD-950023, Computer Science Department, UCLA, 1995.

17. R. D. Skeel. Iterative refinement implies numerical stability for Gaussian elimination. Math. Comput., 35:817-832, 1980.

18. P. E. Strazdins. Issues in the design of scalable out-of-core dense symmetric indefinite factorization algorithms. In Proceedings of the 2003 international conference on computational science: PartIII, ICCS'03, pages 715-724, Springer-Verlag.

19. H. Sutter. The Free Lunch Is Over: A Fundamental Turn Toward Concurrency in Software. Dr. Dobb's Journal, 30(3), 2005. 